Die Qualitätsdiskussion hält Einzug praktisch in jeden Bereich des Gesundheitswesens. Dies ist absolut sinnvoll und nötig, bedarf aber auch konkreter Standards, welche nachvollzogen und kontrolliert werden können. Reine Lippenbekenntnisse reichen heute nicht mehr, damit Patienten, Leistungserbringer, Versicherer und Politik überzeugt werden können. Wer seine Zukunft nicht in die eigene Hand nimmt, wird von anderen geführt.

\title{
Qualität im Rettungswesen - eine komplexe Herausforderung
}

\section{Wolfgang Ummenhofer ${ }^{a}$, Luciano Anselmi ${ }^{b}$, Thomas S. Müller $^{c}$, Gianmaria Solari ${ }^{\text {, }}$ Patrick Siebenpfund ${ }^{e}$, Paul-Martin Sutter ${ }^{\mathrm{f}}$}

${ }^{a}$ Prof. Dr. med., Leitender Arzt Anästhesiologie, Universitätsspital Basel, Präsident Plattform Rettungswesen FMH; b Dr. med., Chefarzt Anästhesie, Regionalspital Bellinzona, Präsident Qualitätskommission IVR; ${ }^{c}$ Dr. med., Chefarzt Zentrale Notfallstation, Kantonsspital Graubünden Chur, Mitglied Swiss Trauma Board; ${ }^{d}$ Dr. med., Lugano, IVR-Vertreter Plattform Rettungswesen FMH; ${ }^{e}$ Dr. med., Basel, SGI-Vertreter Plattform Rettungswesen FMH; f Dr. med., Leitender Arzt Traumatologie Biel, National Director ATLS-Switzerland, SGC-Vertreter Plattform Rettungswesen FMH

Die Plattform Rettungswesen der FMH hat in ihrem 2010 in der Schweizerischen Ärztezeitung erschienenen «Leitbild zum Rettungswesen in der Schweiz» dem Qualitätsmanagement einen besonderen Stellenwert zugewiesen. Der folgende Artikel bezieht sich auf Punkt 8 dieses Leitbilds und soll einzelne Aspekte vertiefen und erläutern.

Für die Strukturqualität des Rettungswesens sind das Einhalten der geforderten Hilfsfristen, die Infrastruktur und die Ausrüstung der involvierten Rettungsdienste, die Einsatzdisposition durch professionell geführte Sanitätsnotrufzentralen, die Ausbildung des eingesetzten Personals, die Auswahl geeigneter Rettungsmittel und der zum Einsatz disponierten Rettungskräfte, insbesondere die Ergänzung der Rettungssanitäter durch den Notarzt bei entsprechender Indikation, die Definition und Selektion geeigneter Zielspitäler und die Qualitätskontrolle der vorgegebenen Standards ausschlaggebend.

- Hilfsfrist für professionelle Rettungsstrukturen: Vital bedrohte Notfallpatienten sollen in der Schweiz innerhalb von 15 Minuten von professionellen Rettungskräften erreicht werden. Wo immer möglich, ist auf eine Hilfsfrist von zehn Minuten hinzuarbeiten, was für Agglomerationen auch eine realistische Option darstellt. Dieses Hilfsfrist-Soll muss die Regelsituation sein - in neun von zehn Fällen muss diese Zeitlimite eingehalten werden. Diese Hilfsfristen gelten sinnvollerweise auch für den Notarzt [1], der bei entsprechender Indikation hinzugezogen werden soll. Allerdings weist die Schweiz trotz dichter Besiedlung geographisch komplexe Regionen auf,

\section{Auf den Punkt gebracht:}

- Notfallpatienten in der Schweiz werden mit einer Hilfsfrist von 15 Minuten von professionellen Rettungskräften versorgt; in Agglomerationen kann in der Regel eine Hilfsfrist von zehn Minuten eingehalten werden.

- Ausgebildete Rettungssanitäter sollen bei entsprechender Indikation durch Notärzte mit FA «Präklinische Notfallmedizin» (Notarzt/Notärztin SGNOR) unterstützt werden.

- Die medizinische Verantwortung jedes schweizerischen Rettungsdienstes liegt beim Ärztlichen Leiter Rettungsdienst, der über einen FA "Präklinische Notfallmedizin» (Notarzt/ Notärztin SGNOR) verfügt.

Notfallpatienten mit speziellen Krankheitsbildern oder Verletzungsmustern sollen direkt in für sie geeignete Zielspitäler transportiert werden; dies ist politisch im Rahmen der HSM-Entscheide für Stroke-, Verbrennungspatienten, Polytrauma oder schweres SHT entschieden worden.

Der IVR ist in der Schweiz die Richtlinien- und Zertifizierungsinstanz für Rettungsdienste und Sanitätsnotrufzentralen.

- Die Weiterbildung zum Notarzt wird von der SGNOR geregelt; ausserhalb der Weiterbildungsinstitutionen sollen Notfallpatienten nur von Notärzten mit gültigem Fähigkeitsausweis versorgt werden.

Für ein sinnvolles Qualitätsmanagement im Rettungswesen werden Outcome-Daten benötigt. Anonymisierte Datenbanken sind für spezielle Patientengruppen im Aufbau. Diese Register sollen institutionell verankert sein und die Daten auf gesamtschweizerischer Basis erheben.

in denen diese Fristen naturgemäss nicht eingehalten werden können. Dort kann ein Dienstarztdispositiv das Notarztnetz ergänzen, was beispielsweise in Graubünden mit kantonaler Unterstützung umgesetzt wird [2]. Lokale Rettungssysteme sollen sich mit überregionalen Rettungsstrukturen vernetzen, 
was dank des effizienten Luftrettungsangebots in unserem Land für die geeigneten Patientengruppen sinnvolle Versorgungsintervalle möglich macht.

- Ärztlicher Leiter Rettungsdienst: Jeder Rettungsdienst in der Schweiz soll medizinisch durch einen Notarzt mit Fähigkeitsausweis «Präklinische Notfallmedizin» (Notarzt/Notärztin SGNOR) geleitet werden. Diese Führungsaufgabe darf keine «Feigenblattposition» sein; der Ärztliche Leiter ist dem nachgeordneten Personal gegenüber in medizinischen Fragen weisungsberechtigt, trägt die Verantwortung für die Delegation ärztlicher Kompetenzen an nichtärztliches Personal und überwacht die medizinische Qualitätssicherung des Rettungsdienstes insgesamt.

\section{Vital bedrohte Notfallpatienten sollen in der Schweiz innerhalb von 15 Minuten von profes- sionellen Rettungskräften erreicht werden.}

- Zielspital: Notfallpatienten sollen nach geeigneter Erstversorgung vor Ort unter kompetenter Überwachung und Behandlung direkt in das für die definitive Versorgung der schwersten Schädigung geeignete Zielspital transportiert werden. Die Kantone haben eine Vereinbarung zur hochspezialisierten Medizin (HSM) unterzeichnet, in der für die Gruppe der Notfallpatienten die Zentren für Stroke, Verbrennungen und Schwerstverletzte explizit definiert sind; anstelle von 26 kantonalen Planungen kommt ein von allen Kantonen gemeinsam getragenes Konzept zur Anwendung. Für die Umsetzung sind ein medizinisch besetztes HSMFachorgan und ein politisch besetztes $H S M-B e$ schlussorgan zuständig. Für die Versorgung von Schwerverletzten wurden zwölf Schweizer Zentrumsspitäler identifiziert, welche Patienten mit einem Injury Severity Score (ISS) von mindestens 20 Punkten und/oder schwere Schädel-HirnTraumata (SHT) behandeln. Die zwölf Traumazentren, die sich im "Swiss Trauma Board» (STB) als einfache Gesellschaft zusammengeschlossen haben, sind mit dem HSM-Entscheid verpflichtet, gemeinsam das Schweizer Traumaregister (STR) zu betreiben und sich für die Entwicklung überregionaler «Trauma-Netzwerke» zu engagieren. Schwierig bleibt zunächst noch die präklinische Triage aufgrund der politisch geforderten Verletzungsschwere für die Versorgung in einem HSM-Traumazentrum. Der ISS als validierter und weitverbreiterter Score in der traumatologischen Literatur ist ein auf anatomische Befunde (z.B. Schnittbildgebung, Operationsbefunde) gestützter Wert, der präklinisch nicht ver- wendet werden kann. Und Zuweisungen aufgrund des Unfallmechanismus oder physiologischer Scores sind wenig sensitiv, was die Gefahr der Unter- respektive Übertriage mit sich bringt. Unabhängig hiervon profitieren Patienten mit einem hohen Mortalitätsrisiko, zu denen die Notfallpatienten mit vitaler Bedrohung zweifelsfrei gehören, von einer optimalen prähospitalen Versorgung und einem strukturierten und etablierten Management im Zentrum. Dies konnte eindrücklich für eine grosse Kohorte mit akutem Koronarsyndrom nachgewiesen werden[3].

- Richtlinien und Empfehlungen: Der Interverband für Rettungswesen (IVR) erlässt im Auftrag der kantonalen Gesundheitsdirektoren Richtlinien für Transportmittel, Ausrüstungen der Rettungsdienste und personelle Besetzung eines Rettungsmittels in Bezug zu Dringlichkeit und vitaler Bedrohung von Notfallpatienten; für entsprechende Indikationen sind ausgebildete Rettungssanitäter bzw. zusätzlich ein Notarzt gefordert. Entsprechende Vorgaben für die Betreiber von Sanitätsnotrufzentralen (SNZ) 144 sollen auch im logistischen Bereich der Einsatzerfassung und -disposition die Rahmenbedingungen für die Schweiz definieren. In Übereinstimmung mit den Reanimationsrichtlinien 2015 sollen die Angehörigen von Patienten mit einem mutmasslichen ausserklinischen Herz-Kreislauf-Stillstand vom SNZ-Disponenten bei ihrem 144-Notruf am Telefon strukturiert in der Durchführung einer

Jeder Rettungsdienst in der Schweiz soll medizinisch durch einen Notarzt mit Fähigkeitsausweis «Präklinische Notfallmedizin» (Notarzt/Notärztin SGNOR) geleitet werden.

mechanischen Laienreanimation instruiert werden [4].

- Qualitätskontrolle, Zertifizierungen: Es ist vorgesehen, dass alle Rettungsdienste in der Schweiz IVR-zertifiziert sind oder eine vergleichbare Qualitätsüberprüfung nachweisen können. Das Anerkennungsverfahren wird vom Rettungsdienst bzw. der SNZ initiiert und nach Vorlage einer Selbstdeklaration durch einen Expertenbesuch überprüft. Der vermehrte Aufwand soll sich für einen zertifizierten Rettungsdienst auch in der Tarifstruktur der Kostenträger widerspiegeln; insofern können qualitätsüberprüfte Strukturen zumindest teilweise finanziell anerkannt werden.

Für die ärztliche notfallmedizinische Weiterbildung ist die Schweizerische Gesellschaft für Notfall- 
und Rettungsmedizin (SGNOR) im Auftrag des SIWF zuständig. Sie überwacht und visitiert die Weiterbildungsstätten für den FA «Präklinische Notfallmedizin" (Notarzt/Notärztin SGNOR) bzw. sogenannte Notarztstandorte, an denen Ärzte in Weiterbildung nach Abschluss des geforderten klinischen Curriculums und nach bestandenen Vorbereitungskursen (ACLS, PALS, Notarztkurs) die notwendigen 50 Notarzt-Einsätze absolvieren können. Ausserhalb dieser Weiterbildungsinstitutionen sollen Notfallpatienten nur von ausgebildeten Notärzten mit FA «Präklinische Notfallmedizin» (Notarzt/Notärztin SGNOR) behandelt werden. Die Qualitätskontrolle für die beiden Kursformate «Notarztkurs» und «Dienstarztkurs» wird von der SGNOR bzw. der Plattform Rettungswesen der FMH sichergestellt; für den Kurs «Sanitätsdienstliche Führung im Grossereignis» (SFG/CSAM) in der Ausbildung zum Leitenden Notarzt (LNA) bzw. Einsatzleiter Sanität (EL San) wird im Rahmen des Projekts «unité de doctrine SFG/CSAM» die Kooperation zwischen dem Koordinierten Sanitätsdienst (KSD) und der SGNOR gegenwärtig neu geregelt. Die entsprechenden Diplome sind befristet und müssen ebenfalls nach fünf Jahren rezertifiziert werden; die hierfür vorgeschriebene Fortbildung bzw. Tätigkeit im Fachgebiet wird in Selbstdeklaration nachgewiesen und stichprobenweise überprüft. Ohne Rezertifizierung verfällt der FA nach sechs Jahren.

- Datenschutzbestimmungen: Ein sinnvolles Qualitätsmanagement ist nur möglich, wenn Rettungseinsätze an ihrer Indikation und Angemessenheit mittels relevanter Outcome-Daten überprüft werden können. Solche Daten existieren bisher in der Schweiz nicht bzw. allenfalls punktuell und regional in Form spezifischer Fragestellungen. Bislang haben ausserdem nachvollziehbare datenschutzrechtliche Gründe solche Datenbanken verhindert. Für die Erstellung von Registern müssen die Persönlichkeitsrechte von Patienten und die rechtlichen
Vorgaben des Datenschutzes gewahrt und dennoch die anonymisierte Erfassung definierter Kenndaten ermöglicht werden. Verschiedene Institutionen, Organisationen und Fachgesellschaften haben mit dem Aufbau solcher Notfalldatenbanken begonnen; wie vom European Resuscitation Council (ERC) angeregt, sollen Kenndaten zu den Notfalldiagnosen des so genannten «First Hour Quintett» gesammelt werden: (1) Herzstillstand, (2) Akutes Koronarsyndrom, (3) Stroke, (4) Akute Atemnot, (5) Polytrauma

Es ist vorgesehen, dass alle Rettungsdienste in der Schweiz IVR-zertifiziert sind oder eine vergleichbare Qualitätsüberprüfung nachweisen können.

bzw. schweres SHT. Erfahrungen aus dem Ausland zeigen, wie schwierig gerade die prähospitale Erfassung und Dokumentation solcher Kenndaten ist und welch kleiner Anteil der klinisch gut dokumentierten Fälle auch eine qualitativ vergleichbare Ausgangsbeschreibung der entsprechenden Werte vor Ort bzw. während des Transports ins Spital ermöglicht. Wenn ein mit viel Aufwand und Kosten verbundenes Register valide ausserklinische Daten erhalten soll, dann ist dies speziell in unserer föderalen Landschaft mit einem sehr heterogenen Rettungswesen nur möglich, wenn sich alle beteiligten Organisationen, Rettungsdienste, SNZ, Spitäler und politische Instanzen, konstruktiv für eine einheitliche Version verwenden.

Literatur

1 Die Gleichstellung von Frau und Mann ist für die Autoren eine Selbstverständlichkeit. Der besseren Lesbarkeit wegen wird im Text meist nur die männliche Form verwendet.

2 Scharplatz D. Der Arzt im Rettungskonzept der Kantone. Schweizerische Ärztezeitung. 2002;83:513-4.

3 Babic D et al. Mortalitätsursachen und Outcome beim akuten Koronarsyndrom. Kardiovaskuläre Medizin. 2015;18:347-54.

42015 American Heart Association Guidelines Update for Cardiopulmonary Resuscitation and Emergency Cardiovascular Care. Circulation. 2015;132,Suppl. 2:397-413. 